\title{
La posture épistémologique de biologistes en lien avec leurs représentations de la démarche scientifique
}

Youssef Boughanmi et Fatma Saïd-Touhami

\section{OpenEdition}

\section{Journals}

Édition électronique

URL : http://journals.openedition.org/trema/3651

DOI : 10.4000/trema.3651

ISSN : 2107-0997

Éditeur

Faculté d'Éducation de l'université de Montpellier

Édition imprimée

Date de publication : 1 décembre 2017

Pagination : 117-128

ISBN : 979-10-96627-03-5

ISSN : 1167-315X

\section{Référence électronique}

Youssef Boughanmi et Fatma Saïd-Touhami, « La posture épistémologique de biologistes en lien avec leurs représentations de la démarche scientifique », Tréma [En ligne], 47 | 2017, mis en ligne le 01 février 2018, consulté le 02 mai 2019. URL : http://journals.openedition.org/trema/3651 ; DOI 10.4000/trema.3651

Ce document a été généré automatiquement le 2 mai 2019.

Trema 


\title{
La posture épistémologique de biologistes en lien avec leurs représentations de la démarche scientifique
}

\author{
Youssef Boughanmi et Fatma Saïd-Touhami
}

\section{Introduction}

1 Plusieurs travaux (Faury, 2013, Kaspar, 2013) ont montré la nécessité d'une interdisciplinarité qui met en tension sciences humaines et sciences techniques. Ces travaux s'interrogent sur l'initiation de la réflexion chez les chercheurs biologistes ; sur la place de l'histoire des sciences et de l'épistémologie dans leurs formations. Si les difficultés institutionnelles d'intégrer les modules des sciences humaines dans la formation des scientifiques ont été très variées, leur enseignement semble se heurter à leur formation de base, leur profession ou leurs recherches. En sciences humaines, les travaux de recherche sont censés apporter un progrès dans la connaissance sous forme d'éclairage sur une question ou une reconstitution d'un corpus explicatif. Ces travaux contiennent, à des proportions différentes, des descriptions et des analyses, mais également un apport personnel de l'auteur. En sciences expérimentales, ces travaux contiennent en plus des propositions de tests de validité (expériences, observations, mesures). De ce fait, il semble pour les apprentis-chercheurs que la biologie, comme patchwork de disciplines, s'identifie (Clement, 1999, Coquidé et Vander, 1998.) par ses frontières qui ont leurs approches spécifiques (biophysique, psychophysiologie biotechnologie, bioinformatique).

2 Par conséquent, l'intégration de modules des sciences humaines dans le programme de biologistes se heurte à la spécificité de la biologie et de ses domaines de recherche. Ors nous avons remarqué dans le cours d'initiation à la recherche (Said, 2007) que des biologistes, même s'ils assimilent les concepts de la démarche scientifique, se trouvent en 
difficulté dans leur application à leurs projets de recherche. La présente étude est un diagnostic de leurs représentations de la démarche scientifique pour prouver l'apport des sciences humaines à leurs cursus de chercheurs, et aussi une réflexion sur la construction de leurs postures de chercheurs. Pour apporter des éléments de réponses à nos questions de recherche, nous présenterons d'abord le module enseigné aux futurs biologistes et nous expliquerons ce que nous entendons par démarche méthodologique. Nous mettrons l'accent aussi sur l'élaboration et l'évolution de la problématique au fur et à mesure de l'avancement du projet tout en réfléchissant sur la temporalité d'une recherche. La partie empirique sera consacrée à un diagnostic des représentations des biologistes de la démarche scientifique et les difficultés qu'ils rencontrent afin de déchiffrer la construction de leur posture de chercheur. Ainsi dans cette étude, nous nous interrogeons sur les difficultés rencontrées par des chercheurs novices dans l'élaboration de leurs projets de recherche, et sur le lien entre ces difficultés et la représentation qu'on ces étudiants chercheurs de la science et de la démarche scientifique. La formation des futurs biologistes portant sur la démarche scientifique pourrait-elle contribuer à la construction de leurs postures de chercheurs?

\section{Contenu du module sur la démarche scientifique}

3 L'enjeu éducatif pour la méthodologie de recherche en sciences humaines semble être intéressant dans la mesure où il peut fournir des instruments opérationnels susceptibles d'aider les étudiants biologistes à élaborer leurs problématiques et par conséquent déterminer le champ de recherche de leurs projets. Cette formation à la démarche méthodologique d'un projet de recherche proposée aux étudiants de mastères biologique à la faculté des sciences de Tunis est une initiation à la recherche qui tente d'expliquer aux étudiants les différentes étapes d'une démarche d'un travail de recherche (Said, 2007). Cette formation commence par montrer aux étudiants les critères d'un sujet scientifique (originalité, faisabilité). Les étudiants sont par la suite invités à proposer un sujet de recherche de leur choix. Une fois le sujet choisi, le formateur accompagne les étudiants dans les différentes démarches de recherche bibliographique, les oriente vers des lectures en relation avec leur projet (lecture active, analytique et d'évaluation), les aide à élaborer la problématique, et à améliorer leur écriture scientifique (formes, étapes, références, ..). L'élaboration de la problématique de recherche, constitue une étape importante de cette formation.

4 Elle consiste à aider les étudiants, chercheurs novices à adopter une posture de chercheur qui part d'un constat d'un problème, pour arriver à se poser des questions pertinentes en lien avec ce problème en vue d'une recherche de solution. La formation met le focus sur ce cheminement. Une dernière partie de la formation porte sur la présentation orale, et aussi la communication des résultats (article, affiche) afin de saisir une autre caractéristique d'une recherche scientifique: la communicabilité et le partage d'informations et des résultats.

\section{La démarche méthodologique}

5 «La relation du vécu de la recherche, désigne le déroulement, le pendant de la recherche, la (re)composition du trajet suivi par le chercheur » (Vial, 1999, p.10). Ce chemin n'est jamais linéaire et monocausal, il n'est pas une trajectoire (Ardoino, 1995), il est processuel, avec 
des allers-retours, des régulations et des régularisations. Entre la démarche méthodologique et le produit final, il y a un travail continu de recueil, d'analyse et d'interprétations, la rédaction et la diffusion peuvent aussi faire partie de ses étapes. Toutefois, ce n'est pas parce que la démarche est processuelle que le produit va l'être, quoiqu'elle nous paraisse personnelle, d'ailleurs, cette démarche n'a jamais été individuelle. La recherche se fait dans un cadre plus large dans une institution et on ne peut pas mener une recherche seul. Dans chaque projet s'impliquent d'autres praticienschercheurs dont leur soutien au chercheur est essentiel dans la construction de sa posture. C'est une situation particulière d'apprentissage (Dubois, 2005) par accompagnement tout en gardant une certaine indépendance de son tuteur ou directeur de recherche.

6 Selon Vial (ibid), la méthodologie est un discours sur les méthodes de recherche, notamment sur la méthode qu'on choisit dans une recherche. L'un des objectifs du discours méthodologique est de rendre communicable l'usage de telle(s) ou telle(s) méthode(s) sans être soucieux de justifier (faire un jugement de valeur pour convaincre) l'utilité d'une technique ou un outil méthodologique. Pour cela, l'investigation méthodologique nécessite une certaine vigilance épistémologique (Bachelard, 1934) pour mener à bien un projet de recherche et permettre le dépassement du sens commun et des préjugés. De ce fait, la démarche méthodologique implique une rupture avec la pensée commune, une problématisation de l'objet de recherche et une vérification par les faits (Boughanmi, 2006). Pour les sciences humaines, mais aussi pour toutes les autres disciplines, la démarche méthodologique met en question la posture épistémologique revendiquée et la méthode employée pour prendre en considération la visée de la recherche (le pourquoi) et son programme (pour obtenir quoi), en fonction notamment du terrain d'investigation mais surtout en fonction du projet à entreprendre.

\section{L'élaboration d'une problématique}

7 La recherche est un investissement qui se base initialement sur les acquis, les pratiques sociales de références, les questions d'autrui...etc. D’une façon générale, un chercheur peut questionner le discours dominant, argumenter des faits ou des données recueillies ou démontrer rationnellement des affirmations (Boughanmi, 2010). C'est un monde de doute qui développe une vision critique et conteste le sens commun tout en discutant sans cesse le choix et le contenu d'un sujet. Il s'agit d'entreprendre, avec une rigueur scientifique, un approfondissement d'une piste de recherches antérieures ou d'explorer des voies nouvelles pour apporter des éléments de réponses aux questions ou hypothèses non validées ou sur un terrain non investigué. L'engagement dans une piste de recherche est basé essentiellement sur la méthodologie de recherche (Grenon et Viau, 2012) qui contribue la construction de la posture du chercheur.

8 Le domaine des sciences biologiques est très vaste et un grand nombre de disciplines peuvent se trouver intéressées, à un moment ou à un autre, par des problèmes de biologie. D'où la difficulté de distinguer ce qui est authentiquement méthodologique vu la diversité des écoles et des laboratoires ainsi que l'innombrable type d'échantillons. Dans une logique méthodologique et suite à un constat ou intérêt personnel, le biologiste détermine son champ d'investigation en fonction de ses lectures qui peuvent être enrichies par des discussions avec des pairs. Le constat ou la littérature débouche sur une esquisse de questions ou une problématique qui conditionnent le choix de la 
méthodologie et des procédures de recueil des données. En fonction de la spécificité du sujet, « la problématique est évolutive, transitoire et située dans le temps » (Vial, ibidem) et elle est souvent construite dans un cadre théorique qui permet de communiquer une problématisation élaborée. "Quand nous rencontrons un problème, il est évident que nous ne pouvons pas en savoir grand-chose. Au mieux, nous n'avons qu'une vague idée de ce en quoi il consiste réellement. Comment alors pouvons-nous élaborer une solution adéquate? (Popper, 1973).

9 La problématisation est donc un processus ou travail engagé pour être en problème, c'est-àdire une situation qui exige une tension entre des éléments qui ne devraient pas aller ensemble et qui pourtant s'imposent dans une même visée (Vial, 1999). La problématique, dans ce sens, est un travail du sujet sur lui-même, elle construit le sujet. C'est parce que le sujet se donne un temps d'articulation des éléments, base du discours problématologique (CIRADE, 1999), que la problématique sera productive. Mais en quoi la construction de la problématique est utile pour forger la posture du chercheur? La problématique ne peut se résumer en une phrase interrogative mais peut faire l'objet d'une question que le sujet pose et se pose.De ce fait, elle peut correspondre à l'ensemble des méthodes ou procédures que le chercheur met en jeu lors du développement de son travail. Cependant, La problématique n'est pas seulement procédurière, elle est aussi épistémologique (CIRADE, 1999). L'interaction entre les méthodes mobilisées pour élaborer et répondre à la problématique d'une recherche permet au chercheur de construire sa posture en lui permettant de dépasser l'opérationnel ; « Savoir comment utiliser ceci ou cela ». La démarche scientifique suivie, ainsi que les allers-retours entre ses différentes étapes, permet au chercheur de déterminer la place de telle méthode dans sa démarche méthodologique et contribue à la construction de sa posture de chercheur.

\section{La temporalité de la recherche et la construction de la posture du chercheur}

10 "Établir un calendrier et suivre un échéancier est essentiel. Il faut au départ de toute recherche estimer avec précision (tout au plus une semaine de marge) le temps que nécessiteront les diverses phases de la recherche " (Albarello, 1999). L'estimation du temps dans une démarche méthodologique reste l'énigme pour la majorité des chercheurs. Il s'agit essentiellement du temps nécessaire à l'organisation temporelle de chaque phase de la recherche. Pour chaque étape existe, presque toujours, un écart entre l'estimation subjective et néophyte du temps nécessaire et sa durée réelle. Il y a un continuum, des allers-retours entre les divers moments de la recherche, mais aussi une relation de type dialectique entre eux. La durée faisant son travail, l'interférence donne un produit acceptable et le chercheur part dans une autre phase. De ce fait, la durée imposée par les organismes reste relative, il y a toujours une marge de semaines voire de mois de retard par rapport au temps estimé. Comme l'affirme Albarello (ibid) certaines informations ne trouvent pas leur sens que dans la durée, il fallait du temps pour l'incubation des idées. Il semble que l'écart entre le temps estimé et le temps réel est dû, principalement, à la non-linéarité des procédures de la recherche (Boughanmi, 2006). Plusieurs autres variables parasites pourraient aussi intervenir: disponibilité des acteurs, contraintes financières, activités parallèles (par exemple profession, famille, etc. La scientificité de la recherche exige la rigueur méthodologique, comme elle nécessite du temps. Quel que soit la recherche entreprise, et dans tous les domaines, la durée ne peut que jouer un rôle important dans la construction 
de la posture du chercheur, et ce par le biais de la démarche scientifique spécifique de chaque discipline.

\section{Recueil et analyse des données}

11 L'enquête a été menée par l'enseignant chargé du module méthodologie de recherche, dont le contenu est décrit précédemment durant l'année universitaire. Il s'agit d'un questionnaire de neuf questions ouvertes pour permettre aux étudiants de mobiliser le maximum d'informations. Les questions portent sur deux catégories d'items : la première catégorie porte sur la représentation de la science par les étudiants et la deuxième catégorie porte plutôt sur les difficultés de la mise en œuvre d'une démarche scientifique dans un projet. Les questions élaborées reflètent le contenu cours enseigné. L'échantillon représente une population hétérogène d'étudiants en 1re année mastère sciences biologiques est composé de 57 étudiants répartis dans des filières en fonction de leurs choix (tableau 1). S'ils réussissent la première année, ils entament un essai de recherche sous la direction d'un chercheur et fera l'objet d'un mémoire de Mastère.

Tableau I : répartition des étudiants selon les mastères

\begin{tabular}{|l|c|}
\hline Mastères & Effectif en \% \\
\hline Biotechnologie végétale & $21 \%$ \\
\hline Biologie intégrative des écosystèmes littoraux & $22 \%$ \\
\hline Ecophysiologie vègétale & $28 \%$ \\
\hline Génétique et biosciences & $28 \%$ \\
\hline Ecologie végétale & $1 \%$ \\
\hline
\end{tabular}

Pour analyser les données recueillies, nous avons procédé à une catégorisation des questions réponses pour répondre à nos objectifs d'étude. Nous lisons l'ensemble des réponses et nous regroupons dans des catégories les représentations proches. Les réponses qui renferment plus qu'une idée ou opinion peuvent être classées dans deux ou plusieurs catégories (Q2, Q6). Les questions 5 et 7 étant formées deux parties, les réponses sont donc classées séparément (tableau 3). Dans un premier temps, nous analysons les réponses aux questions portant sur les représentations des chercheurs biologistes de la science et la démarche scientifique. Nous nous focalisons par la suite sur l'analyse des questions servant plutôt à diagnostiquer les difficultés qui peuvent entraver l'avancement du projet de recherche des chercheurs biologistes.

\section{1. Les représentations de la science par des chercheurs biologistes}

13 Nous récapitulons dans le tableau suivant l'ensemble des catégories des réponses aux questions 1 à 4 . Les catégories de la première question au cadre de la recherche effectué par l'étudiant. 
Tableau 2 : représentations des étudiants de la science

\begin{tabular}{|c|c|c|}
\hline Questions & Catégories & Effectif \% \\
\hline \multirow{5}{*}{$\begin{array}{l}\text { QI : est-ce que vous avez fait déjà une recherche } \\
\text { scientifique ? Si oui, dans quel cadre? }\end{array}$} & Biotechnologie médicale & $3 \%$ \\
\hline & Technologie & $1 \%$ \\
\hline & Ingénieur horticole & $1 \%$ \\
\hline & Jamais & $87 \%$ \\
\hline & Non précisé & $13 \%$ \\
\hline \multirow{4}{*}{$\begin{array}{l}\text { Q2 : Pouvez-vous donner une définition de la } \\
\text { uscience » }\end{array}$} & Ensemble de savoirs & $43 \%$ \\
\hline & Vitalisme & $22 \%$ \\
\hline & Anthropomorphisme & $22 \%$ \\
\hline & Explication & $35 \%$ \\
\hline \multirow{3}{*}{$\begin{array}{l}\text { Q3 : Proposez un exemple de théorie } \\
\text { scientifique et un exemple d'autre théorie? } \\
\text { Justifiez pour chaque exemple la scientificité } \\
\text { ou non de chaque théorie. }\end{array}$} & $\begin{array}{l}\text { Théorie Scientifique : } \\
\text { ensemble de lois }\end{array}$ & $22 \%$ \\
\hline & $\begin{array}{l}\text { Théorie scientifique } \\
\text { évolutions des techniques }\end{array}$ & $29 \%$ \\
\hline & $\begin{array}{l}\text { Théorie non scientifique : } \\
\text { Droit de l'homme } \\
\text { Théorie de l'évolution }\end{array}$ & $49 \%$ \\
\hline \multirow{4}{*}{$\begin{array}{l}\text { Q4 : Proposez des phénomènes aux quels la } \\
\text { science ne peut donner des explications. } \\
\text { Pour quelle (s) raison (s)? }\end{array}$} & $\begin{array}{l}\text { Phénomène : explication } \\
\text { divine (la mort, l'origine } \\
\text { de la vie) }\end{array}$ & $49 \%$ \\
\hline & $\begin{array}{l}\text { Phénoméne : la science } \\
\text { essaye d'expliquer } \\
\text { (neurologie, fonction } \\
\text { des organes) }\end{array}$ & $45 \%$ \\
\hline & Scientologie & $1 \%$ \\
\hline & Pas de réponses & $5 \%$ \\
\hline
\end{tabular}

14 La majorité $(87 \%)$ des étudiants interrogés dans cette enquête n'ont jamais fait de la recherche. Néanmoins ils sont habitués à manipuler aux laboratoires dans le cadre des travaux pratiques. A ce stade, la démarche expérimentale acquise se limite au plan habituel de travail: hypothèse observation, expérience, résultats, interprétation et conclusion. Alors qu'un sujet de recherche trouve ses origines dans la littérature ou dans la société. Avec de nouveaux concepts de la démarche scientifique, le formateur se trouve face à des apprenti-chercheurs dont leur définition de la science elle-même est prête à confusion. Même la théorie de l'évolution, à laquelle adhère la majorité des scientifiques depuis la révolution darwinienne, est considérée comme une théorie non scientifique par $49 \%$ des biologistes. Les théories scientifiques regroupent plutôt les lois et les technologies. On retrouve chez certains étudiants des explications qui renvoient au vitalisme, la science permet d'expliquer la vie d'une façon générale. La moitié des chercheurs biologistes pensent que la science ne peut pas expliquer la mort ou l'origine de la vie. Le vitalisme, ce courant scientifique qui date des années trente, est dépassé actuellement, et la question de la vie relève plutôt du domaine de la philosophie. D'autres étudiants renvoient la science à l'anthropomorphisme vu que cette science est au service de l'homme, ce narcissisme limite le rôle explicatif de la science au sens large. Cette explication est scientifique que $35 \%$ du public la mobilise semble être limitée au corps humain (neurologie, fonction des organes). Nous remarquons aussi que les verbes utilisés par les étudiants renvoient à la démarche expérimentale avec ses différentes étapes (hypothèse, problématique), et par conséquent entravent l'acquisition de certains concepts de méthodologie de recherche (élaboration de problématique par exemple). Certains étudiants ont évoqué l'argumentation scientifique sans approfondir leurs propos.

15 L'explication en biologie, même si elle fait appel à la physique ou d'autres disciplines, oscille entre une biologie fonctionnaliste et une biologie historique. Cette dernière ne se limite pas à des lois (Gayon, 1993) mais plutôt aux exigences de son historicité. En relation 
directe avec le laboratoire dans lequel le chercheur mène ses recherches, l'usage des appareils et des outils techniques pour mesurer, calculer ou manipuler, la définition d'un domaine scientifique peut dépendre de l'évolution technologique. L'échange mutuel entre sciences et technologies permet d'améliorer la fiabilité et la validité de la recherche. La non-scientificité de la théorie de l'évolution est liée essentiellement à son cadre historique et à l'absence de travaux pratiques dans ce type de cours.

Les droits de l'homme ne sont pas considérés par les étudiants comme une théorie scientifique. Cette hiérarchisation des disciplines est due à un cloisonnement, primo, institutionnel vu que cette science est enseignée à la faculté des lettres alors que la biologie s'enseigne à la faculté des sciences, et secundo, l'absence de laboratoire de recherche au sens des biologistes. Une théorie scientifique ne doit pas sa scientificité à la terminologie "science " mais plutôt aux méthodes spécifiques à chaque domaine scientifique. La libération de l'esprit par la sagesse ou scientologie, comme courant très récent ( $\mathrm{XX}^{\mathrm{e}}$ siècle), risque-t-elle de développer un certain dogmatisme scientifique ? Le parallélisme explication divine et explication scientifique, répandu dans les réponses des étudiants, peut-il limiter l'apport explicatif de la science ? L'initiation des chercheurs biologistes à la démarche scientifique permet la rupture, même partielle, avec les préjugés et limite chez eux la hiérarchisation sociale des domaines scientifiques. La construction de la posture épistémologique (Chabchoub, 1998) du chercheur nécessite l'interaction perpétuelle entre plusieurs domaines connexes de son projet de recherche.

\section{2. Les difficultés de l'élaboration d'un projet de recherche}

17 Nous récapitulons dans le tableau suivant la catégorisation de réponses aux questions 5 à 8. Les deux parties de la question 7 sont catégorisés séparément pour identifier les difficultés des étudiants à déterminer les caractéristiques de la problématique et de sa construction.

\begin{tabular}{|c|c|c|}
\hline Questions & Catégories & Effectif\% \\
\hline \multirow{4}{*}{$\begin{array}{l}\text { Q5: } \\
\text { - Proposez un sujet de } \\
\text { recherche dans votre discipline. }\end{array}$} & Génie génétique & $17 \%$ \\
\hline & Problèmes environnementaux & $24 \%$ \\
\hline & Biotechnologie & $31 \%$ \\
\hline & Santé & $28 \%$ \\
\hline \multirow{3}{*}{$\begin{array}{l}\text { - Quels sont vos critères de } \\
\text { choix pour ce sujet? }\end{array}$} & Critères économiques & $21 \%$ \\
\hline & $\begin{array}{l}\text { Nécessité humaine } \\
\text { (santé surtout) }\end{array}$ & $61 \%$ \\
\hline & Critères écologiques & $38 \%$ \\
\hline \multirow{5}{*}{$\begin{array}{l}\text { Q6: } \\
\text { A partir de la recherche que } \\
\text { vous avez déjà effectuée dans le } \\
\text { module de } \\
\text { méchodologie de recherche } \\
\text { cette année, avez-vous } \\
\text { rencontrés des difficultés? }\end{array}$} & Choix du sujet & $36 \%$ \\
\hline & $\begin{array}{l}\text { Recherche bibliographique et } \\
\text { sitographique }\end{array}$ & $37 \%$ \\
\hline & Elaboration de la problématique & $40 \%$ \\
\hline & Difficultés de rédaction & $28 \%$ \\
\hline & Difficultés lors de l'exposé oral & $29 \%$ \\
\hline \multirow{6}{*}{$\begin{array}{l}\text { Q7: } \\
\text {-Qu'est-ce que caractérise une } \\
\text { problelematique }\end{array}$} & $\begin{array}{l}\text { Intérêt du sujet : économique, } \\
\text { politique, scientifique }\end{array}$ & $12 \%$ \\
\hline & Répond à un objectif précis & $29 \%$ \\
\hline & Découverte et curiosité & $5 \%$ \\
\hline & Les questions de recherche & $17 \%$ \\
\hline & $\begin{array}{l}\text { Etudier un problème réel et } \\
\text { actuel }\end{array}$ & $14 \%$ \\
\hline & Résolution d'un problème & $21 \%$ \\
\hline \multirow{6}{*}{$\begin{array}{l}\text { - Comment peut-on construire } \\
\text { une problématique ? }\end{array}$} & Présentation du sujet & $29 \%$ \\
\hline & Questions de recherche & $33 \%$ \\
\hline & Sujet : Cause / effet & $8 \%$ \\
\hline & Hypothèses & $7 \%$ \\
\hline & Recherche des données & $5 \%$ \\
\hline & Recherche bibliographique & $1 \%$ \\
\hline \multirow{3}{*}{$\begin{array}{l}\text { Q8: Comment vérifiez-vous } \\
\text { la fiabilité et la validité des } \\
\text { données dans les sujets de } \\
\text { recherche que vous avez } \\
\text { proposée? }\end{array}$} & Recherche bibliographique & $52 \%$ \\
\hline & Démarche expérimentale & $33 \%$ \\
\hline & $\begin{array}{l}\text { Recours aux référents: } \\
\text { encadreur, jury, collègues }\end{array}$ & $28 \%$ \\
\hline
\end{tabular}


18 Le choix des sujets reste dans le cadre des filières de la biologie auxquelles sont inscrits les étudiants et touchent des domaines très récents en biologie tels que l'environnement, les OGM.

Les critères de choix des sujets sont principalement économiques, et écologiques mais la majorité des étudiants voient en leurs sujets une réponse à des nécessités humaines. $61 \%$ des étudiants travaillent dans cadre explicatif anthropomorphique, la biologie quand elle ne peut se limiter as seulement au service de l'homme.

20 À ce stade de recherche, l'étudiant n'a pas fait assez de lecture dans la littérature qui traite son sujet. D'ailleurs la majorité des étudiants trouvent des difficultés de faire des recherches bibliographiques ou sitographiques. Pour certains étudiants la problématique est l'équivalent du titre du projet et ils trouvent des difficultés pour développer les questions de recherche. La rédaction scientifique s'acquiert en fonction de l'avancement de la recherche et l'incubation des idées prend du temps. La rédaction scientifique est devenue une nécessité pour les tous les novices et ils ont précisé qu'ils trouvent des difficultés pour exposer oralement, surtout au début d'une communication. Nous retenons chez certains étudiants que la problématique dépend de la curiosité et la découverte alors que pour d'autres elle dépend des questions permettant le développement d'un problème réel et actuel. La lecture mais aussi le constat mènent à la reformulation de questions ou à la construction d'autres qui peuvent induire le fonctionnement de l'élaboration de la problématique. L'intérêt du sujet, la réponse à un objectif ou la résolution d'un problème dépendent de la démarche générale du projet de recherche. Un seul étudiant a renvoyé la problématique aux références bibliographiques, les hypothèses et le recueil des données font partie de la démarche expérimentale. La présentation du sujet devant les pairs ou la discussion avec l'encadrant permet d'éclaircir les pistes de travail et on peut parler d'une ébauche de problématique. Pour la plupart des étudiants interrogés, la problématique est limitée aux questions de recherche. Ils ont du mal à relier les questions de recherches de leur propre travail à l'état d'avancement des travaux antérieurs sur le même problème.

21 Si la recherche bibliographique ne constitue pas la base de l'élaboration scientifique, elle est essentielle pour la majorité des étudiants pour valider des données. Pour les étudiants, et contrairement à la démarche scientifique, les références font partie des dernières phases du projet alors qu'elles sont la base pour élaborer la problématique. La démarche expérimentale permet la validation des données, si on peut retrouver les mêmes résultats dans une recherche comparable. Bien évidemment, l'encadreur et le jury valident le travail mais sa diffusion reste un outil de validité et de fiabilité. Selon Chalmers (1987), à travers la démarche inductive, la vérité des données qui aboutissent aux théories et lois, est favorisée par une observation attentive. Lorsque les conditions pour procéder à des inductions légitimes sont réunies (observations répétées dans des conditions différentes, nombre suffisant de données ou d'énoncés d'observation), les lois et les théories seront à leurs tours aussi sûres que les observations. En fait, les relations entre significations qui constituent la connaissance scientifique sont exprimées par des propositions (Dewey, 1983), qui ne peuvent pas être qualifiées de vraies ou de fausses mais de valides ou d'invalides. Selon Meyer (1979), qui adhère à une conception rationaliste ou problématologique de la science, les réponses se réfèrent obligatoirement à des problèmes. Par conséquent, elles ne sont ni vraies ni fausses, mais qui conviennent ou non comme réponse au problème. 


\section{Conclusion} disciplines scientifiques, a montré qu'il y a des difficultés d'ordre épistémologique, des difficultés d'ordre méthodologique et d'autres difficultés liées à la discipline elle-même, ici la biologie. Les difficultés d'ordre méthodologique concernent surtout la littérature et la synthèse des documents et l'organisation du travail. L'élaboration de la problématique est une difficulté majeure. En effet, la plupart des étudiants ont du mal à distinguer un problème - qui existe déjà - d'une problématique qui est construite par le chercheur à partir du problème préexistant et l'état d'avancement des recherches antérieurs sur ce problème. La rédaction du mémoire lui-même, et au-delà des difficultés techniques liées aux références, aux normes, pose problème surtout au niveau de la rédaction de l'introduction et de la conclusion. Ces deux parties très importantes dans le mémoire, par rapport au message que porte chacune, sont difficiles à développer par de nombreux étudiants. Leurs différences, ainsi leur complémentarité sont des éléments clés à saisir par l'étudiant. Nous avons identifié aussi chez les étudiants des obstacles épistémologiques. Ils se réfèrent au sens commun pour élaborer des problèmes scientifiques. La majorité de leur réponse font de l'être humain le principal acteur au service de qui nous faisons la recherche, cet anthropomorphisme limite la diversité des champs de recherche. Dans certaines réponses, nous avons retrouvé des explications qui renvoient au vitalisme et au matérialisme, courants scientifiques que la biologie ellemême les a dépassé au profit de nouvelles doctrines explicatives. La difficulté liée à la recherche en biologie, comme patchwork de disciplines, est de distinguer ce qui est authentiquement méthodologique vu la diversité des écoles et des laboratoires ainsi que l'innombrable type d'échantillons. Le recours à la religion, pour répondre à certaines questions, nécessite un approfondissement théorique, mais aussi un travail de terrain.

Ce travail a montré qu'une représentation principalement techniciste et utilitariste de la science, explique bel et bien le recours des étudiants de mastères biologiques au choix de sujets liés plutôt aux problèmes environnementaux, sanitaires. Leur difficulté majeure dans l'élaboration de leurs projets de recherche est liée à leur représentation d'une science basée exclusivement sur le constat de problèmes, l'observation, la recherche de données quantifiées. L'adhésion complète à cette démarche, dite scientifique, éloigne ces apprentis-chercheurs d'une étape importante dans l'élaboration de leurs projets de recherche : celle de la prise en compte des recherches antérieures sur le même problème. Pour ces étudiants, la consultation des travaux de recherche à travers la recherche bibliographique, vient surtout alimenter la discussion, vérifier la validité de certaines données, ... donc a posteriori. Une formation en sciences humaines sur des questions épistémologiques et sur les différents types de démarches de recherche, semble être indispensable pour montrer aux futurs chercheurs que la science se construit dans un processus de rupture et de continuité. Ceci est un levier pour aider les étudiants à pallier la difficulté de construire une vraie problématique de recherche, qui est loin d'être un simple problème constaté ou observé. Travailler sur la représentation de la science et de la démarche scientifique par des chercheurs biologistes est un exemple de l'utilité des sciences humaines dans la formation des scientifiques. Cette formation favorise la mise en œuvre de projets scientifiques. Dans ce sens la nécessité d'une formation sur la démarche scientifique, ancré dans une réflexion épistémologique, est primordiale, pour en donner 
sens au projet entrepris, ici par des biologistes, et contribue à la construction de leur posture épistémologique en tant que futurs chercheurs.

\section{BIBLIOGRAPHIE}

Albarello L. (1999). Apprendre à chercher. De Boeck et Larcier.

Ardoino J. (1995). Editorial. L'année de la recherche n², 1995, pp 5-8.

Bachelard G. (1934). Le nouvel esprit scientifique. PUF. 7e édition « quadrige » : 2008, février.

Boughanmi Y. (2010). Cours de méthodologie quantitative et qualitative. Maison des sciences de l'homme, Université de Rouen. Non publié.

Boughanmi Y. (2006). Formaliser une démarche méthodologique sous l'ancre de la temporalité. Colloque international sur la didactique, la pédagogie et la relation université - environnement. Médéa, Algérie, 14-15 Mai.

CIRADE. Les sciences de l'éducation en question. Revue consacrée aux réflexions, aux recherches et aux études sur des pratiques éducatives abordées depuis des problématiques d'apprentissage et/ ou de didactique et/ou d'évaluation. Les cahiers de l'année 1999.

Chabchoub A. (1998). Les didactiques des disciplines : enjeux épistémologiques, institutionnels et éducatifs. Communication au symposium international de didactique des disciplines. Gammarth 1998.

Chalmers A.-F. (1987). Qu'est-ce que la science ? Éditions la découverte. Pour la traduction française.

Clément P. (1999). Les spécificités de la biologie et de son enseignement. Biologie géologie, 3.

Coquidé Cantor M., Vander Borght C. (1998). Des recherches en didactique de la biologie : finalités, problématiques, concepts et productions. Thèmes, thèses, tendances. Aster 27. INRP.

Dewey J. (1983 [1977]). La réalité comme expérience. Trad. Saint Grenier P et Truc G (2005). ENS.

Dubois J.-M. (2005). La rédaction scientifique. Mémoires et thèses; formes régulière et par articles. Editions Estem.

Faury M. (2013). Initier la démarche réflexive chez les futurs chercheurs en biologie expérimentale. Colloque sciences humaines en sciences et techniques. Créteil 7-8 février 2013.

Gayon J. (1993). La biologie entre loi et histoire. Philosophie, les éditions de minuits, 38, pp 50-57.

Kaspar C. (2013). L'histoire des sciences, l'épistémologie, et la didactique dans la formation des étudiants en Master spécialité métiers de l'enseignement des sciences physiques : modalités, contenus et problématiques. Colloque sciences humaines en sciences et techniques. Créteil 7-8 février 2013.

Meyer E. (1979). Découvertes et justification en sciences. Klincksied. Paris.

Grenon G., Viau S. (2012). Méthodes quantitatives en sciences humaines. De l'échantillon vers la population. $4 \mathrm{e}$ éditions. Chenelière Education. 
Popper K. (1973). La logique de la découverte scientifique. Paris : Payot (éd. originale : Londres, 1959).

Said F. (2007). Cours de méthodologie de recherche. Faculté des sciences de Tunis. Non publié.

Vial M. (1999). « Ceci n'est pas un lexique : 25 mots pour la recherche en sciences de l'éducation » In les sciences de l'éducation en question, cahiers $n^{\circ} 25$. CIRADE. Université de Provence, AixMarseille I.

\section{RÉSUMÉS}

Dans le cadre de la préparation d'un projet de recherche, les étudiants tunisiens de mastères biologiques suivent une formation d'initiation à la recherche et à la démarche scientifique. Dans ce papier, nous essayons de mettre l'accent sur la place des sciences humaines, représentée ici par la démarche scientifique, dans la construction de la posture épistémologique des futurs biologistes. Donc nous interrogeons leurs représentations de la démarche scientifique afin de mieux comprendre le lien entre leurs représentations et les difficultés qu'ils rencontrent dans l'élaboration de leurs projets de recherche. Le diagnostic montre que les difficultés sont plurielles et sont liées, principalement, à une représentation techniciste et utilitariste de la science.

To prepare their research project, Tunisian students of biological master Degree undergo an initiation in research and scientific method. In this paper, we try to focus on the place of the human sciences, represented here by the scientific method in the construction of the epistemological posture of future biologists. Therefore, we analyze their misconceptions s of the scientific process to understand the link between these representations and the difficulties they encounter in developing their research projects. The diagnosis shows that the difficulties are related to a technicist and utilitarian representation of science.

\section{INDEX}

Mots-clés : représentations, démarche scientifique, posture épistémologique, chercheurs biologistes

Keywords : misconception, scientific methods, epistemological posture, biologists

\section{AUTEURS}

\section{YOUSSEF BOUGHANMI}

Université de Bourgogne, Centre Georges Chevrier

FATMA SAÏD-TOUHAMI

ESPE Aix-Marseille, AE ADEF 